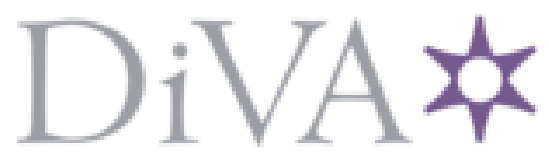

http://www.diva-portal.org

This is the published version of a paper presented at IEEE PES ISGT Europe.

Citation for the original published paper:

Rogersten, R., Vanfretti, L., Li, W., Zhang, L., Mitra, P. (2014)

A Quantitative Method for the Assessment of VSC-HVdc Controller Simulations in EMT Tools.

In:

N.B. When citing this work, cite the original published paper.

Permanent link to this version:

http://urn.kb.se/resolve?urn=urn:nbn:se:kth:diva-161751 


\title{
A Quantitative Method for the Assessment of VSC-HVdc Controller Simulations in EMT Tools
}

\author{
Robert Rogersten ${ }^{1}$, Luigi Vanfretti ${ }^{1,2}$, Wei $\mathrm{Li}^{1}$, Lidong Zhang ${ }^{3}$, and Pinaki Mitra ${ }^{3}$ \\ ${ }^{1}$ KTH Royal Institute of Technology, Sweden \\ ${ }^{2}$ Statnett SF, Norway \\ ${ }^{3} \mathrm{ABB}$, Sweden
}

\begin{abstract}
Voltage-Source Converter-Based High-voltage dc (VSC-HVdc) technology has received growing interest during recent years due to its benefits for separated active and reactive power control. Before the installation of VSC-HVdc links, different simulation-based studies need to be performed, usually by different parties and using different electromagnetic transient (EMT) simulation platforms. This paper provides a systematic method to assess discrepancies between simulation tools. Models of the VSC and its control systems are implemented from scratch in two different tools. A metric and a methodology to quantitatively assess the discrepancies between simulation results is proposed and illustrated. The paper shows that even with a pedantic re-implementation of the models from the ground up, simulation results are still inconsistent. The objective of this paper is to develop a quantification method for comparing the models developed in different EMT simulation tool and to emphasize differences that can arise with the numerical solvers in such tools.
\end{abstract}

Index Terms-VSC-HVdc modeling and control, EMT simulation tools, Quantitative assessment method, PSCAD, Simulink

\section{INTRODUCTION}

\section{A. VSC-HVdc Technology and Simulation Studies}

$\mathrm{H}$ IGH-VoLTAGE direct current (HVdc) systems using voltage source converters (VSC) have been attracting increasing attention since the turn of the century [1]. One reason for this is the increase in electricity consumption throughout the world. In Europe alone, total electricity consumption has increased by $32.8 \%$ from 1990 to 2007. As a result, the European high-voltage alternating-current (HVac) grid is operating close to its physical limits [2]. In order to facilitate interconnections within an ac grid, VSC-HVdc interconnections could be embedded in a conventional ac grid and provide a strong backbone to existing ac networks, as done in the past with conventional HVdc links [3], but with increased ability to address issues regarding power transmission, asynchronous network interconnections, and stability support [4].

VSC-HVdc systems offer unique operational flexibility thanks to different control systems. However, the design and tuning of these controls for each installation needs to be assessed using simulation tools prior to installation. In addition, research on new control methods and techniques also requires the use of simulation tools prior to prototype implementation to reduce development costs.

To perform these studies, electromagnetic transient (EMT) models of the VSC and its control systems are required. A critical issue arises when the model of the VSC-HVdc is available only for a specific tool. This constraints a systematic assessment of the VSC-HVdc design and controller performance by different parties; which is particularly critical when a transmission system operator (TSO) needs to perform studies in tool A and the VSC-HVdc models from the manufacturer are only available in tool $\mathrm{B}$, which opens the door for ambiguity. Today's EMT tools do not offer the facility for model exchange [5], which leads to differences in simulation results between tools. This opens the door for speculation on the 'correctness' of the model or the adequacy of a simulation tool. Further, these speculations are made based on a visual inspection of simulation outputs and without a systematic methodology for assessment. Hence, it is clear that a baseline on the assessment of simulation results needs to be drawn, and this requires a quantitative method.

\section{B. Contributions}

To address issues discussed above, this paper provides a systematic methodology to assess discrepancies between EMT simulation tools. To this aim, a VSC and generic converter controller models are pedantically implemented using two different EMT modeling and simulation platforms. A metric and a methodology to quantitatively assess the discrepancy between simulation results from two different platforms is described and illustrated using the aforementioned models. As this paper shows, the discrepancies between software models are not acceptable even when a pedantic re-implementation of the model 'from the ground up' has been carried out in each tool.

\section{Converter Modeling And Control}

In this paper, PSCAD and MATLAB/Simulink have been used to simulate a point-to-point VSC-HVdc system. PSCAD and Simulink are graphical tools that can be used for EMT simulation of electric power systems.

It is possible to model a VSC, either in detail or by using an average value model (AVM). If a VSC is modeled in detail all semiconductor components such as IGBTs can be included as a single unit represented in the model [6]. However, such detailed modeling approach increases simulation time compared to an AVM; therefore, an AVM which represents the average response of the converter by using controlled 


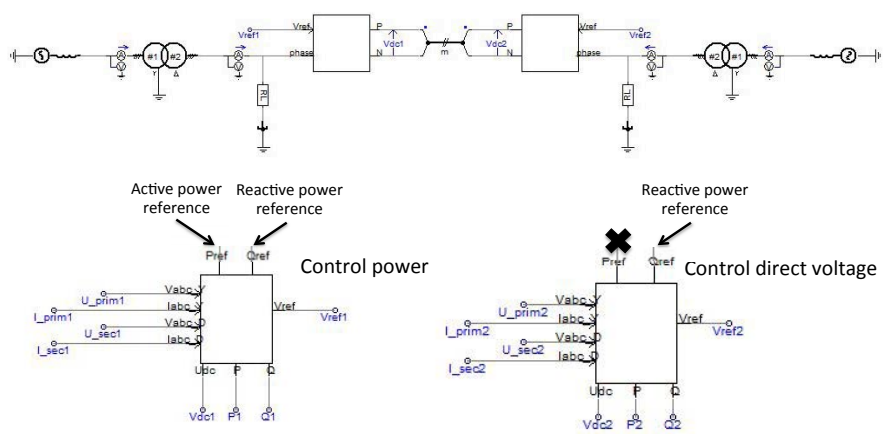

Fig. 1. Schematic of the point-to-point VSC-HVdc link model in PSCAD

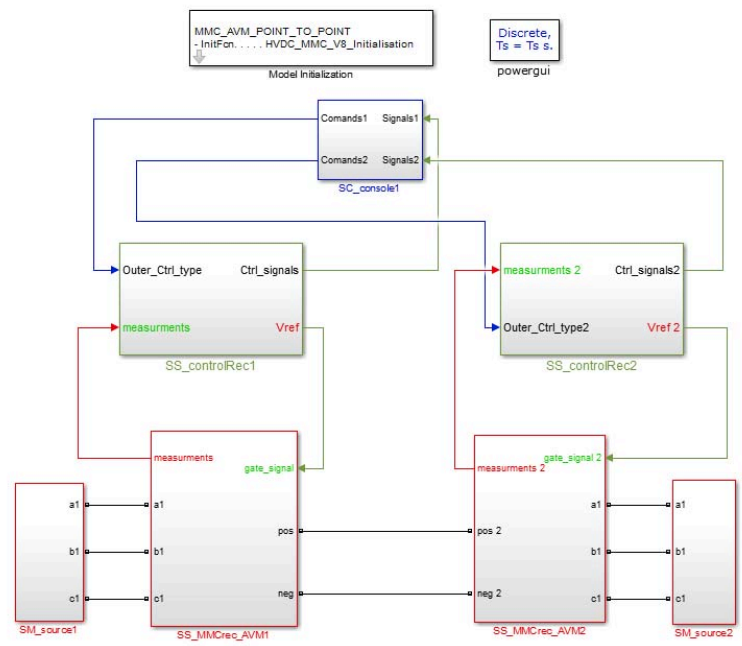

Fig. 2. Schematic of the point-to-point VSC-HVdc link model in Simulink

sources and averaged functions is used in this work. The AVM implementations in PSCAD and Simulink are based on the model proposed in [7]. Schematics of PSCAD and Simulink are shown in Fig. 1 and Fig. 2, respectively.

\section{A. Vector-Current Control}

The control strategy used in this paper is commonly referred to as vector-current control, which has been successfully applied on many actual VSC-HVdc link installations. Furthermore, there are many design approaches for the vector-current control strategy. The vector-current control strategy is illustrated in Figure 3. In this section, a common design approach of vector-current control, often referred to as Diagonal Internal Model Control (DIMC) [8], [9], is discussed.

In DIMC, the three-phase currents and voltages are transformed to $d$ and $q$ axes, which makes the fundamental current and voltages become dc components. Therefore, PI-controllers can be used to reduce steady state errors. The final step is to transform the $d$ and $q$ voltages to three-phase quantities.

The relationship between the converter current $\mathbf{i}_{d q}=$ $\left(\begin{array}{ll}i_{d} & i_{q}\end{array}\right)^{T}$, the bridge ac voltage $\mathbf{v}_{d q}=\left(\begin{array}{ll}v_{d} & v_{q}\end{array}\right)^{T}$, and the ac line voltage $\mathbf{u}_{d q}=\left(\begin{array}{ll}u_{d} & u_{q}\end{array}\right)^{T}$, in the dq-plane is

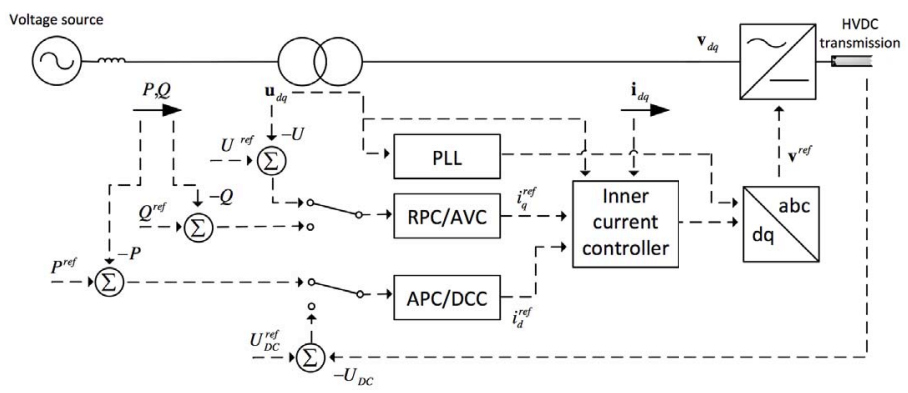

Fig. 3. Main circuit including the control block diagram for vector-current control. The blocks include the phase-locked loop (PLL), reactive-power controller (RPC), alternating-voltage controller (AVC), active-power controller (APC) and dc-controller (DCC).

$$
\mathbf{v}_{d q}=\mathbf{u}_{d q}+\omega_{1} L\left(\begin{array}{c}
i_{q} \\
-i_{d}
\end{array}\right)-L \frac{d \mathbf{i}_{d q}}{d t}-r \mathbf{i}_{d q},
$$

where $\omega_{1}$ is the angular frequency of the ac system, $L$ is the leakage inductance of the transformer, and $r$ is the interconnecting resistance. The resistance $r$ in high voltage applications is usually small and therefore neglected. Therefore, an approximation of each element of $\mathbf{v}_{d q}$ in (1) gives

$$
\begin{aligned}
& v_{d}=u_{d}+\omega_{1} L i_{q}-L \frac{d i_{d}}{d t}, \\
& v_{q}=u_{q}-\omega_{1} L i_{d}-L \frac{d i_{q}}{d t} .
\end{aligned}
$$

The system is obviously coupled. Therefore, a decoupler is added to an inner feedback loop of the system. By letting

$$
\begin{aligned}
& v_{d}=u_{d}+v_{d}^{\prime}+\omega_{1} L i_{q}, \\
& v_{q}=u_{q}+v_{q}^{\prime}-\omega_{1} L i_{d},
\end{aligned}
$$

the decoupled system becomes

$$
\begin{aligned}
& v_{d}^{\prime}=-L \frac{d i_{d}}{d t}, \\
& v_{q}^{\prime}=-L \frac{d i_{q}}{d t} .
\end{aligned}
$$

The transfer function from $\mathbf{v}_{d q}^{\prime}$ to $\mathbf{i}_{d q}$ is therefore given by

$$
G_{d}(s)=\left(\begin{array}{cc}
-\frac{1}{s L} & 0 \\
0 & -\frac{1}{s L}
\end{array}\right) .
$$

The system represented by (5) is controlled using a cascade control structure. Therefore, an inner control loop and an outer control loop are designed.

\section{B. Vector-Current Control Inner Loop}

The transfer function in (5) is decoupled and can therefore be controlled with a diagonal PI-controller, which means that the $d$ and $q$ components can be controlled independently as two single-variable systems. An illustration of the decoupling is shown in Figure 4. The PI-controller can be expressed as

$$
F_{\mathrm{PI}}(s)=-\left(\begin{array}{cc}
k_{p}+\frac{k_{i}}{s} & 0 \\
0 & k_{p}+\frac{k_{i}}{s}
\end{array}\right) .
$$




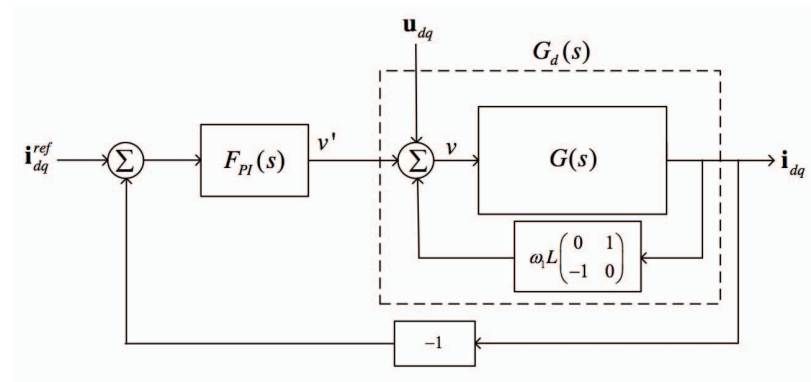

(a) Block-diagram of the feedback loop. $F_{\mathrm{PI}}(s)$ is the diagonal PIcontroller and $G(s)$ is the system transfer function.

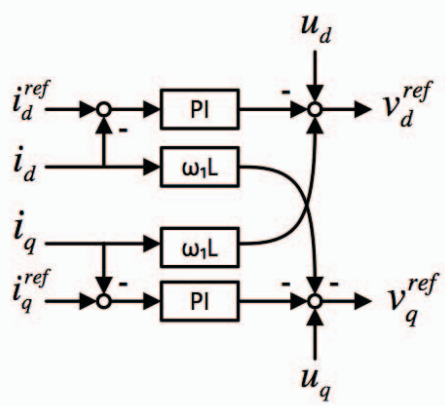

(b) Detailed diagram that illustrates the separate PI-controllers and the reference voltages. The separate PI-controllers are given by PI $=k_{p}+\frac{k_{i}}{s}$.

Fig. 4. The inner control loop of vector-current control. The inner control loop has an inner decoupling. Basically, Figure $4 \mathrm{a}$ and $4 \mathrm{~b}$ are two ways to represent the same controller. However, Figure $4 \mathrm{a}$ gives a better overview of the diagonal transfer function $G_{d}(s)$.

The decoupled system has a negative transfer function; therefore, the PI-controller is implemented with a minus sign.

For some implementations, a low pass filter $H_{\mathrm{LP}}(s)$ is added to the control law to improve disturbance rejection.

Assuming $\mathbf{v}_{d q}^{\text {ref }}=\mathbf{v}_{d q}$ yields the following control law

$$
\mathbf{v}_{d q}^{\mathrm{ref}}=\left(\begin{array}{c}
u_{d} \\
u_{q}
\end{array}\right)+F_{\mathrm{PI}}(s)\left(\begin{array}{c}
i_{d}^{\mathrm{ref}}-i_{d} \\
i_{q}^{\mathrm{ref}}-i_{q}
\end{array}\right)+\omega_{1} L\left(\begin{array}{c}
i_{q} \\
-i_{d}
\end{array}\right)
$$

where the references $i_{d}^{\text {ref }}$ and $i_{q}^{\text {ref }}$ are given by an outer control loop. The current control in (7) is referred to as the inner control loop.

\section{Vector-Current Control Outer Loop}

The outer control loop feeds the reference current to the inner control loop in order to maintain an adequate reference voltage for the VSC. Depending on the mode of operation, the reference $i_{d}^{\text {ref }}$ is used to control the active power or direct voltage. In the same way, the reference $i_{q}^{\text {ref }}$ is used to control the reactive power or ac voltage. There are several ways to calculate the reference currents. In this paper, an integral controller with feed-forward is used. When the activeand reactive powers are controlled, the reference currents are calculated as

$$
\mathbf{i}_{d q}^{\mathrm{ref}}=\frac{1}{V}\left(\begin{array}{c}
P_{\mathrm{ref}}+\frac{k_{i}}{s}\left(P_{\mathrm{ref}}-P\right) \\
-Q_{\mathrm{ref}}-\frac{k_{i}}{s}\left(Q_{\mathrm{ref}}-Q\right)
\end{array}\right)
$$

where $V=\left|\mathbf{v}_{d q}\right|=\sqrt{v_{d}^{2}+v_{q}^{2}}$ is the voltage magnitude at the converter bridge.

As an alternative to the traditional PI-controller, [10] explored the properties of an IP-controller and showed some advantages compared to the PI-controller for implementations on dc drives. In order to control alternating and direct voltages, models in this paper utilizes IP-controllers. Using an IPcontroller, the reference currents for direct- and alternating voltages are calculated as

$$
\mathbf{i}_{d q}^{\mathrm{ref}}=\left(\begin{array}{c}
\frac{k_{i}}{s}\left(U_{\mathrm{dc}}^{\mathrm{ref}}-U_{\mathrm{dc}}\right)-k_{p} U_{\mathrm{dc}} \\
\frac{k_{i}}{s}\left(U^{\mathrm{ref}}-U\right)-k_{p} U
\end{array}\right),
$$

where $U=\left|\mathbf{u}_{d q}\right|=\sqrt{u_{d}^{2}+u_{q}^{2}}$ is the voltage magnitude at the primary side of transformer and $U_{\mathrm{dc}}$ is the direct voltage. If preferred, it is also possible to control the voltage at the ac converter bridge instead of primary side of transformer by replacing $U$ with $V$. In order to improve disturbance rejection a low pass filter $H_{\mathrm{LP}}(s)$ can be added for the controllers in (8) and (9).

In addition to (8) and (9), there are two more control modes. If the system is configured to control the active power and the ac voltage, the reference currents are calculated as

$$
\mathbf{i}_{d q}^{\mathrm{ref}}=\left(\begin{array}{c}
\frac{1}{V}\left[P_{\mathrm{ref}}+\frac{k_{i}}{s}\left(P_{\mathrm{ref}}-P\right)\right] \\
\frac{k_{i}}{s}\left(U^{\mathrm{ref}}-U\right)-k_{p} U
\end{array}\right),
$$

and if the system is configured to control the direct voltage and the reactive power, the reference currents are calculated as

$$
\begin{gathered}
\mathbf{i}_{d q}^{\mathrm{ref}}=\left(\begin{array}{c}
\frac{k_{i}}{s}\left(U_{\mathrm{dc}}^{\mathrm{ref}}-U_{\mathrm{dc}}\right)-k_{p} U_{\mathrm{dc}} \\
\frac{1}{V}\left[-Q_{\mathrm{ref}}-\frac{k_{i}}{s}\left(Q_{\mathrm{ref}}-Q\right)\right]
\end{array}\right) . \\
\text { III. COMPARISON AND ANALYSIS }
\end{gathered}
$$

This section compares and analyzes different software implementations of vector-current control. The Simulink model will be considered as a reference for all comparisons. As this section will show, the discrepancies between software models are not acceptable.

The comparisons are performed both graphically and numerically. First, the methodology for numerical comparisons is described. Then, the fault impedance calculation and the system configuration during tests are described. Finally, Section III-C presents numerical and graphical comparisons.

\section{A. Quantitative Assessment Metric and Methodology}

The root mean square (RMS) of the difference between simulation outputs is proposed as a quantitative assessment metric. Numerical comparisons are performed between the two models, using the active- and reactive power simulation outputs of each implementation. The RMS value is calculated according to

$X_{\mathrm{RMS}}=\sqrt{\left.\frac{1}{n}\left[\left(x_{1}-y_{1}\right)^{2}+\left(x_{2}-y_{2}\right)^{2}+\cdots+\left(x_{n}-y_{n}\right)^{2}\right]\right)}$,

where $x_{i}$ is the discrete measurement point at time $t_{i}$ for software (a), and $y_{i}$ is the discrete measurement point at time $t_{i}$ for software (b). That is, if $x(t)$ and $y(t)$ are continuous 
TABLE I

TEST SCENARIOS USED FOR CONTROLLER PERFORMANCE COMPARISONS

\begin{tabular}{c|c}
\hline Test \# & Explanation of test scenario \\
\hline $\mathbf{1}$ & Step of the active-power controller by $\mathbf{3 0 \%}$ \\
2 & Step of the reactive-power controller by $30 \%$ \\
$\mathbf{3}$ & Three-phase fault with 10\% remaining voltage \\
4 & Three-phase fault with 30\% remaining voltage \\
5 & Three-phase fault with 70\% remaining voltage \\
6 & Single-phase fault with $10 \%$ remaining voltage \\
7 & Single-phase fault with 30\% remaining voltage \\
8 & Single-phase fault with 70\% remaining voltage \\
\hline
\end{tabular}

Note: Faults are injected for $100 \mathrm{~ms}$. Scenarios in bold correspond to the graphical results in Fig. 5 and Fig. 6.

TABLE II

RMS CALCULATIONS ACCORDING TO (12)

\begin{tabular}{ccccc}
\hline Test scenario & 1 & 2 & 3 & 4 \\
\hline$P_{\text {RMS }}$ & 0.0030 & 0.0144 & 0.0435 & 0.0386 \\
$Q_{\text {RMS }}$ & 0.0143 & 0.0026 & 0.0628 & 0.0573 \\
\hline \hline Test scenario & 5 & 6 & 7 & 8 \\
\hline$P_{\text {RMS }}$ & 0.0421 & 0.0557 & 0.0542 & 0.0208 \\
$Q_{\text {RMS }}$ & 0.0513 & 0.0587 & 0.0555 & 0.0081 \\
\hline
\end{tabular}

functions of the active- or reactive power, then $x\left(t_{i}\right)=x_{i}$ and $y\left(t_{i}\right)=y_{i}$, where $t_{1}<\ldots<t_{i}<\ldots<t_{n-1}<t_{n}$. The RMS value is calculated on an interval of $1.5 \mathrm{~s}$ divided into 15000 measurement points (i.e., $t_{i+1}-t_{i}=0.0001$ ). The interval is chosen for a duration when the perturbation is applied in the middle. For instance, if a fault is applied at $t=1 \mathrm{~s}$, then $t_{1}=0.5 \mathrm{~s}$ and $t_{15000}=1.9999 \mathrm{~s}$.

\section{B. Fault Impedance and System Configuration}

The generators voltage is set to $U=275 \angle 0^{\circ} \mathrm{kV}$ and they operate at $50 \mathrm{~Hz}$. Each generator has an impedance of $Z_{\text {gen }}=16.9 \angle 85^{\circ} \Omega$, which yields a short-circuit capacity of approximately $S_{\mathrm{ac}}=4500 \angle 85^{\circ} \mathrm{MVA}$. The HVdc link is set to $300 \mathrm{MW}$. It is possible to calculate a fault impedance $Z_{f}$ that yields a desired remaining voltage with the generator voltage and the system short-circuit capacity. Let $n$ denote the fraction of remaining voltage during fault (e.g., $n=0.1$ represents $10 \%$ remaining voltage). The calculation is given by

$$
I_{f}\left(Z_{\mathrm{gen}}+Z_{f}\right)=U \Longrightarrow\left\{\begin{array}{c}
I_{f} Z_{\text {gen }}=n U \\
I_{f} Z_{f}=(1-n) U
\end{array} .\right.
$$

It follows that

$$
\left.\begin{array}{c}
Z_{\mathrm{gen}}=\frac{|U|^{2}}{S_{\mathrm{ac}}^{*}} \\
\frac{Z_{\mathrm{gen}}}{Z_{f}}=\frac{n}{1-n}
\end{array}\right\} \Longrightarrow Z_{f}=\frac{|U|^{2}}{S_{\mathrm{ac}}^{*}} \frac{(n-1)}{n}=\frac{|U|^{2}}{\left|S_{\mathrm{ac}}\right|} \frac{(n-1)}{n} \angle 85^{\circ},
$$

where $S_{a c}^{*}$ denotes the complex conjugate of $S_{\mathrm{ac}}$.

\section{Controller Tests}

Figure 5a illustrates a comparison of the active power during a three-phase fault with $10 \%$ remaining voltage. For the same test scenario, the reactive power is shown in Figure 5b. Further, Figure 6 shows a step response of the active power. In total, eight test scenarios have been performed, which are listed in Table I. Figure 5 and 6 illustrates test scenario 1 and 3. Further, table II illustrates the numerical analysis for all test scenarios, which are calculated according to (12). Note that test scenario 1 and 2 own the best overall matches. Furthermore, the reactive powers have better matches than active powers in test scenario 2 and 8 . In all the other test scenarios, the active powers match better than the reactive powers.

The conclusion from Figs. 5 and 6, and RMS calculations, is that the simulations do not have an overall satisfactory match. The means (here denoted $\bar{X}$ ) of all active- and reactive power RMS measurements are

$$
\begin{aligned}
& \bar{P}_{\mathrm{RMS}}=0.0340, \\
& \bar{Q}_{\mathrm{RMS}}=0.0388 .
\end{aligned}
$$

\section{CONCLUSIONS AND FURTHER WORK}

It does not take much effort to implement models graphically in PSCAD. However, for unambiguous modeling and simulation, one drawback with the standard PSCAD library components is that it is difficult to know which mathematical model is implemented within the graphical block. The phaselocked loop (PLL) can be used to illustrate this issue. If a replica of the PLL in Simulink is applied in PSCAD, it is difficult to know which are the implementation differences. Therefore, when a model is graphically built in PSCAD, its behavior does not make a perfect match to the Simulink model. This paper has suggested a metric and methodology to quantitatively assess differences in simulation outputs between PSCAD and Simulink.

In order to eliminate the ambiguity in results from different simulation tools, it is necessary to minimize (15) and (16). On-going work shows that these values can be significantly reduced. The controls in PSCAD can be tailored to replicate the behavior of Simulink by using automatically generated $\mathrm{C}$ code. This approach achieves 4 times lower value of (15) and 33 times lower value of (16) for the specific test scenarios listed in Table I. These results will be further illustrated in another publication. In addition, this approach does not significantly increase the simulation time. Figure 7 illustrates the active power differences for Scenario 3 using this approach, which provides a higher consistency of simulation results between different platforms.

\section{ACKNOWLEDGMENTS}

The authors gratefully acknowledge Mr. S. Dennetiere and Dr. H. Saad of RTE, France, for the provision of VSC-HVdc models.

The economical support of the institutions and funding bodies listed below is sincerely acknowledged:

- $\mathrm{ABB}$, Sweden;

- EIT KIC InnoEnergy through Action 2.1 of the Smart Power project;

- The EU funded FP7 iTesla project;

- Statnett SF, the Norwegian Transmission System Operator; and

- The STandUP for Energy collaboration initiative. 


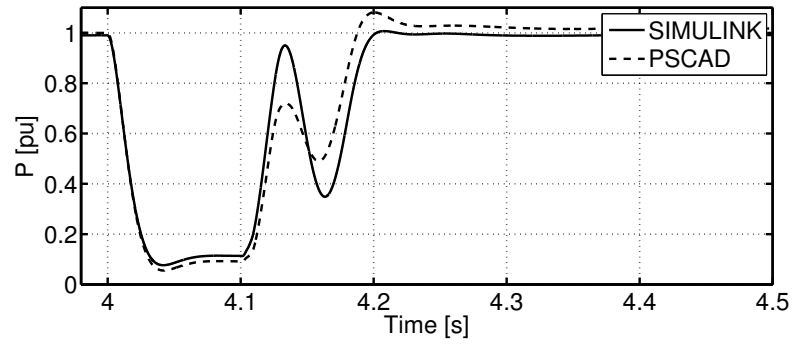

(a) Active-power measurement.

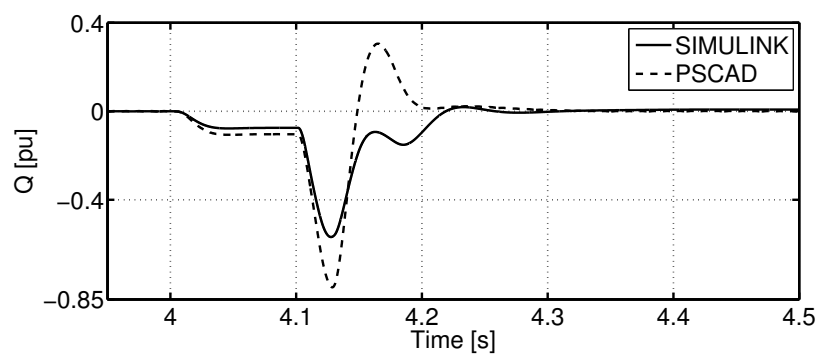

(b) Reactive-power measurement.

Fig. 5. Illustration of the powers during a three-phase fault with $10 \%$ remaining voltage. Figure $5 \mathrm{a}$ presents the active-power and Figure $5 \mathrm{~b}$ the reactive-power. Note that axes have different ratio for Figure 5a and Figure 5b.

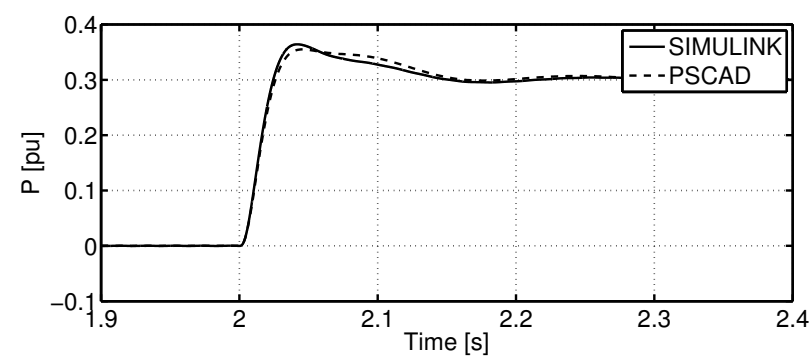

(a) Active-power measurement.

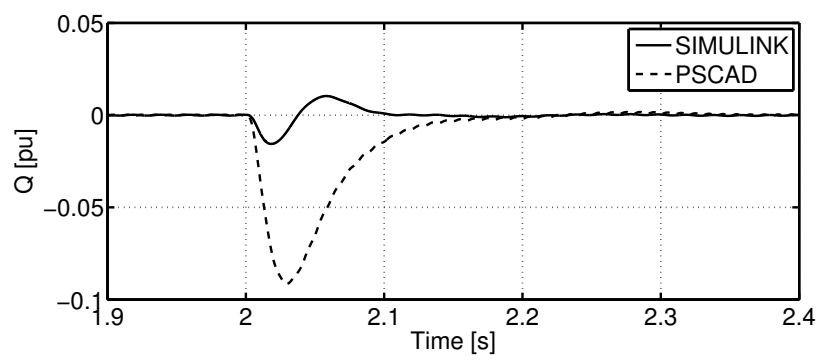

(b) Reactive-power measurement.

Fig. 6. Active-power step change by $30 \%$ at $t=2 \mathrm{~s}$. Figure $6 \mathrm{a}$ illustrates the change in active power and Figure $6 \mathrm{~b}$ illustrates the reactive power response due to the change in active power. Note that axes have different ratio for Figure $6 \mathrm{a}$ and Figure $6 \mathrm{~b}$.

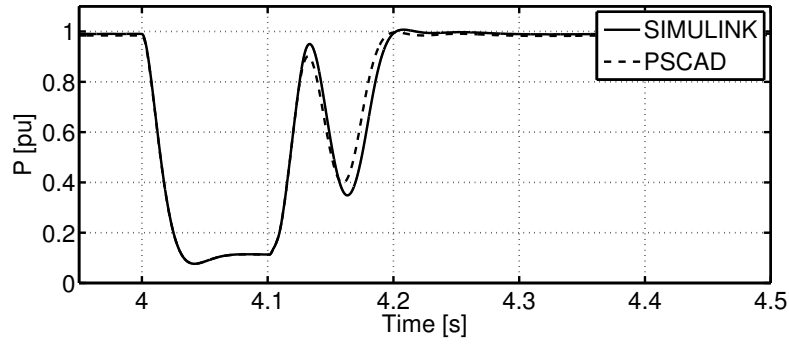

(a) Active-power measurement.

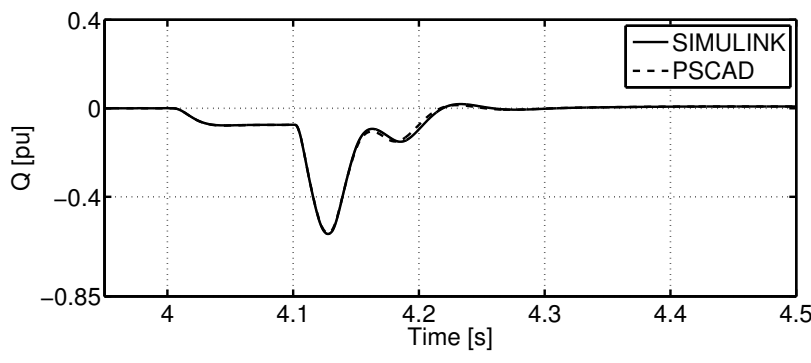

(b) Reactive-power measurement.

Fig. 7. Illustration of when the alternative approach is used. The power is shown during a three-phase fault with $10 \%$ remaining voltage. Figure $7 \mathrm{~b}$ represent the active-power and Figure $7 \mathrm{~b}$ represent the reactive-power. Note that axes have different ratio for Figure $7 \mathrm{~b}$ and Figure $7 \mathrm{~b}$. Ratios are the same as in Figure 5 .

\section{REFERENCES}

[1] N. Flourentzou, V. Agelidis, and G. Demetriades, "VSC-Based HVDC Power Transmission Systems: An Overview," IEEE Transactions on Power Electronics, vol. 24, no. 3, pp. 592-602, 2009.

[2] W. Sattinger, "Application of PMU measurements in Europe TSO approach and experience," in IEEE Trondheim PowerTech 2011, June 2011, pp. $1-4$.

[3] A. Hammad and W. Kuhn, "A computation algorithm for assessing voltage stability at ac/dc interconnections," IEEE Transactions on Power Systems, vol. 1, no. 1, pp. 209-215, Feb 1986.

[4] D. Mueller, M. Rabe, and W. Kuehn, "Stabilizing control for HVDC connected offshore wind farm," in 2013 4th IEEE/PES Innovative Smart Grid Technologies Europe (ISGT EUROPE), Oct 2013, pp. 1-5.

[5] E. Lambert, X. Yang, and X. Legrand, "Is CIM suitable for deriving a portable data format for simulation tools?" in 2011 IEEE Power and Energy Society General Meeting, July 2011, pp. 1-5.

[6] L. Vanfretti, N. A. Khan, W. Li, M. Hasan, and A. Haider, "Generic VSC and low level switching control models for offline simulation of VSCHVDC systems," in 2014 Electric Power Quality and Supply Reliability Conference $(P Q)$, June 2014, pp. 265-272.

[7] J. Peralta, H. Saad, S. Dennetiere, J. Mahseredjian, and S. Nguefeu, "Detailed and averaged models for a 401-level MMC-HVDC system," Power Delivery, IEEE Transactions on, vol. 27, no. 3, pp. 1501-1508, 2012.

[8] L. Harnefors and H.-P. Nee, "Model-based current control of AC machines using the internal model control method," IEEE Transactions on Industry Applications, vol. 34, no. 1, pp. 133-141, 1998.

[9] L. Zhang, "Modeling and control of VSC-HVDC links connected to weak ac systems," Ph.D. dissertation, KTH, Electrical Machines and Power Electronics, 2010, qC20100607.

[10] P. K. Nandam and P. Sen, "Analog and digital speed control of dc drives using proportional-integral and integral-proportional control techniques," Industrial Electronics, IEEE Transactions on, vol. IE-34, no. 2, pp. 227 233, 1987. 